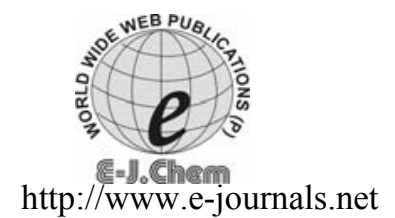

IIIIIIIIII

ISSN: 0973-4945; CODEN ECJHAO

E-Journal of Chemistry

2011, 8(S1), S7-S12

\title{
Synthesis of Novel Dinaphthosulfide, Aza, Oxa, Thia Lariats Containing Urea and Thiourea Groups in the Side Chain and Their Nanostructures
}

\author{
E. ROSTAMI ${ }^{*}$ and A. ZARE
}

Faculty of Science, Department of Chemistry, Bushehr Payame Noor University P.O. Box 1698, Bushehr, Iran; Payame Noor University (PNU), Iran esmrostami@yahoo.com

Received 30 August 2010; Accepted 24 November 2010

\begin{abstract}
Dinaphhosulfide aza, oxa, thia crown was prepared; its urea and thiourea derivatives were synthesized by the reaction with ethyl and 4-chlorophenyl isocyanate, ethyl and phenylisothiocyanate. Self-assembled structures are as micro and nano fibers and discs.
\end{abstract}

Keywords: Aza oxa thia crown, Synthesis, receptor, Macrocycle, Lariat, Nano and Microstructures

\section{Introduction}

Crwon ethers were reported in 1967 by Pedersen for the first time ${ }^{1,2}$. The chemistry of crown ethers has been developed in four decades by a lot of research groups ${ }^{3-6}$. The first crown ethers are all oxa crowns but modification of their properties have been performed using other heteroatoms such as $\mathrm{N}$ and $\mathrm{S}$ in their rings and addition of groups on the rings ${ }^{7}$. Modified crown ethers and aza crowns are compounds bearing various kinds and number of oxa, aza and thia heteroatom in their structures $^{8}$. The second strategy for the modification of crown ethers and their derivatives is the synthesis of crown compounds with different structures, such as cryptands, calixarenes, lariats and biscrowns ${ }^{9-12}$.

Crown ethers and their derivatives are important role in host-guest chemistry and supramolecular chemistry ${ }^{13}$. The complexation properties of these compounds towards metal ions were reported by pedersen ${ }^{1}$ and other researchers were investigated and explored the complexation and interaction of these compounds towards metal ions and neutral or ionic molecules and groups ${ }^{14}$. Noncovalent interactions and 
bonds are the main interactions in host-guest and supramolecular chemistry ${ }^{15}$. These interactions led to the molecular aggregations and these studies are active route in chemical researches in recent years ${ }^{16}$. Nanotechnology is mainly based on the supramolecular chemistry and noncovalent interactions. A lot of natural or synthetic crown ether derivatives are an important role in nanosciences ${ }^{17}$.

In this research work, a new dinaphthosulfide oxa, aza, thia crown was prepared and its urea and thiourea derivatives were synthesized using a series of isocyanate and isothiocyanate derivatives. The aggregations of these macrocycles were studied by scanning electron microscopy using their SEM images. SEM images show the aggregations of molecules based on the noncovalent interactions as the micro and nano discs and fibres.

\section{Experimental}

The reactions were carried out in an efficient hood cupboard. All the materials were purchased from Merck, Fluka, Across Organics and Aldrich chemical companies. Methanol and $\mathrm{CH}_{2} \mathrm{Cl}_{2}$ were distilled and stored under Lind $4 \AA$ molecular sieve. The melting points (uncorrected) were measured with an Electrothermal engineering LTD 9100 apparatus. Elemental analyses were performed by a CHN-O- Rapid Heraeus elemental analyzer. IR spectra were measured on a Perkin-Elmer model 543 , the ${ }^{1} \mathrm{H}$ NMR and ${ }^{13} \mathrm{C}$ NMR spectra were obtained using BRUKER AVANCE DPX $300 \mathrm{MHz}$ and BRUKER AVANCE DRX $500 \mathrm{MHz}$ apparatus. Mass spectra were obtained with Shimadzu GC-MS-QP 1100 EX model. Scanning electron micrograph (SEM) images were obtained using a XL30 (Philips) apparatus.

\section{General procedure for the synthesis of lariats}

To aza crown $(3,1 \mathrm{mmol})$ in $\mathrm{CH}_{2} \mathrm{Cl}_{2}(50 \mathrm{ml})$ was added triethylamine $(1 \mathrm{mmol})$ and then isocyanates (isothiocyanates) $(1 \mathrm{mmol})$. The resulting mixtures were stirred at roon temperature for 24 hours. After completion of the reactions (monitored by TLC), water was added and the resulting reaction mixture was extracted with $\mathrm{CH}_{2} \mathrm{Cl}_{2}(3 \times 30 \mathrm{~mL})$. The combined organic layer were washed with dillute hydrochloric acid twice, dried $\left(\mathrm{Na}_{2} \mathrm{SO}_{4}\right)$ and evaporated to afforded crude products which was purified by recrystallization in chloroform.

Synthesis of 7,10,13-triaza-1-thia-4,16-dioxa-6,14-dioxo-10-(2-aza-1-thio3-phenylethyl)-2,3; 17,18-dinaphtho-cyclooctadecane (8)

This compound was prepared from the reaction of $\mathbf{3}$ and phenylisothiocyanate based on the general procedure, afforded $\mathbf{8}$ as a white powder in the yield of $94 \%$ and melting point of $201-202{ }^{\circ} \mathrm{C}$. IR (KBr): 3396, 3387, 3299, 3052, 2936, 1688, $1679,1623,1558,1543,1446,1323,1265,1226,1153,1077,763 \mathrm{~cm}^{-1} ;{ }^{1} \mathrm{H}$ NMR (300 MHz, DMSO-d $\left.\mathrm{d}_{6}\right) \delta: 3.49$ (broad, 4H), $3.62(\mathrm{~s}, 4 \mathrm{H}), 4.46(\mathrm{~s}, 4 \mathrm{H}), 7.24-7.46$ (m, 11H), $7.86-7.95(\mathrm{~m}, 4 \mathrm{H}), 8.09$ (s, broad, 1H), 8.33 - 8.40 (m, broad, $2 \mathrm{H}), 8.77$ (s, broad, 1H), 9.11 (s, broad, $1 \mathrm{H}) \mathrm{ppm} . ;{ }^{13} \mathrm{C}$ NMR $\left(75 \mathrm{MHz}, \mathrm{DMSO}-\mathrm{d}_{6}\right.$ ) $\delta: 169.39$, $163.10,155.67,143.36,134.05,129.99,129.31,128.62,128.38,127.32,126.99$, $126.09,124.09,124.05,116.50,114.41,67.19,64.92,54.68,53.47,48.66,33.90$ ppm.; MS (EI) m/z: 501 (initial macrocycle (3), 2\%), 500 (1\%), 434 (4\%), 376 (3\%), 318 (1\%), 300 (21\%), 216 (26\%), 202 (24\%), 187 (42\%), $144(81 \%), 115$ (100\%), 93 (24\%), 77 (12\%), 63 (28\%), 39 (27\%); Anal. Cald. for $\mathrm{C}_{35} \mathrm{H}_{32} \mathrm{~N}_{4} \mathrm{O}_{4} \mathrm{~S}_{2}$ : C, $66.02 \%$; H, 5.07\%; N, 8.80\%; Found : C, 66.18\%; H, 5.01\%; N, 8.94\%. 
Synthesis of 7,10,13-triaza-1-thia-4,16-dioxa-6,14-dioxo-10-(2-aza-1-thiobuthyl)-2,3; 17,18-dinaphtho-cyclooctadecane (9)

This larite was prepared from the reaction of ethylisothiocyanate and 3 based on the general procedure and afforded 9 in $87 \%$ yield and melting point of $191-192{ }^{\circ} \mathrm{C}$. IR (KBr): 3395, 3297, 3059, 2925, 1678, 1542, 1503, 1432, 1265, 1236, 1089, 1076, $814, \mathrm{~cm}^{-1} ;{ }^{1} \mathrm{H}$ NMR (300 MHz, DMSO- $\left.\mathrm{d}_{6}\right) \delta: 3.37-3.39(\mathrm{~m}, 4 \mathrm{H}), 3.76(\mathrm{dd}, 4 \mathrm{H}$, $\mathrm{J}=3,6 \mathrm{~Hz}), 4.06(\mathrm{dd}, 2 \mathrm{H}, \mathrm{J}=3,6 \mathrm{~Hz}), 4.50(\mathrm{~s}, 4 \mathrm{H}), 4.94(\mathrm{dd}, 1 \mathrm{H}, \mathrm{J}=2,10 \mathrm{~Hz})$, $5.03(\mathrm{dd}, 1 \mathrm{H}, \mathrm{J}=3,16 \mathrm{~Hz}), 5.70-5.81(\mathrm{~m}, 1 \mathrm{H}), 7.32-7.42(\mathrm{~m}, 6 \mathrm{H}), 7.61(\mathrm{t}, 1 \mathrm{H}$, $\mathrm{J}=6 \mathrm{~Hz}), 7.86-7.93(\mathrm{~m}, 6 \mathrm{H}), 8.27(\mathrm{dd}, 2 \mathrm{H}, \mathrm{J}=3,7 \mathrm{~Hz}) \mathrm{ppm} ;{ }^{13} \mathrm{C}$ NMR $(75 \mathrm{MHz}$, DMSO $\left.-\mathrm{d}_{6}\right) \delta: 169.90,155.60,130.85,130.66,129.94,128.55,128.48,127.96$, $125.38,125.16,124.99,119.95,113.74,112.92,68.60,68.11,53.00,49.90,48.49$, 39.24, $37.55 \mathrm{ppm}$; MS (EI) $\mathrm{m} / \mathrm{z}: 501$ [initial macrocycle (3)] ${ }^{+}(4 \%), 500(2 \%), 483$ (2\%), 300 (8\%), 218 (6\%), 187 (9\%), 144 (100\%), 115 (62\%), 56(5\%), 28 (16\%); Anal. Cald. for $\mathrm{C}_{31} \mathrm{H}_{32} \mathrm{~N}_{4} \mathrm{O}_{4} \mathrm{~S}_{2}$ : C, 63.24\%; H, 5.48\%; N, 9.52\%; Found : C, $63.18 \%$; H, 5.53\%; N, 9.65\%.

Synthesis of 7,10,13-triaza-1-thia-4,16-dioxa-6,14-dioxo-10-(2-aza-1-oxo3-(4-chlorophenyl)-ethyl)-2,3; 17,18-dinaphtho-cyclooctadecane (10)

This compound was prepared from the reaction of $\mathbf{3}$ and 4-chlorophenylisocyanate according to the general procedure and afforded 10 in $88 \%$ yield and melting point of $221-222{ }^{\circ} \mathrm{C}$. IR (KBr): 3396, 3299, 3058, 2928, 1674, 1624, 1540, 1505, 1445 , $1380,1327,1268,1245,1086,1077,813 \mathrm{~cm}^{-1} ;{ }^{1} \mathrm{H}$ NMR (300 MHz, DMSO-d 6 ) $\delta: 3.37-3.39(\mathrm{~m}, 4 \mathrm{H}), 3.76(\mathrm{dd}, 4 \mathrm{H}, \mathrm{J}=3,6 \mathrm{~Hz}), 4.06(\mathrm{dd}, 2 \mathrm{H}, \mathrm{J}=3,6 \mathrm{~Hz}), 4.50$ $(\mathrm{s}, 4 \mathrm{H}), 4.94(\mathrm{dd}, 1 \mathrm{H}, \mathrm{J}=2,10 \mathrm{~Hz}), 5.03(\mathrm{dd}, 1 \mathrm{H}, \mathrm{J}=3,16 \mathrm{~Hz}), 5.70-5.81$ $(\mathrm{m}, 1 \mathrm{H}), 7.32-7.42(\mathrm{~m}, 6 \mathrm{H}), 7.61(\mathrm{t}, 1 \mathrm{H}, \mathrm{J}=6 \mathrm{~Hz}), 7.86-7.93(\mathrm{~m}, 6 \mathrm{H}), 8.27(\mathrm{dd}$, $2 \mathrm{H}, \mathrm{J}=3,7 \mathrm{~Hz}) \mathrm{ppm} ;{ }^{13} \mathrm{C}$ NMR $\left(75 \mathrm{MHz}, \mathrm{DMSO}-\mathrm{d}_{6}\right) \delta: 169.90,155.60,130.85$, $130.66,129.94,128.55,128.48,127.96,125.38,125.16,124.99,119.95,113.74$, $112.92,68.60,68.11,53.00,49.90,48.49,39.24,37.55$ ppm; MS (EI) $\mathrm{m} / \mathrm{z}: 501$ [initial macrocycle (3)] $]^{+}(3 \%), 500(1 \%), 483(2 \%), 300(8 \%), 212(6 \%), 187(7 \%)$, $144(100 \%), 115$ (62\%), 44 (16\%); Anal. Cald. for $\mathrm{C}_{35} \mathrm{H}_{31} \mathrm{ClN}_{4} \mathrm{O}_{5} \mathrm{~S}: \mathrm{C}, 64.16 \% ; \mathrm{H}$, $4.77 \%$; N, 8.55\%; Found : C, 64.07\%; H, 4.83\%; N, 8.63\%.

Synthesis of 7,10,13-triaza-1-thia-4,16-dioxa-6,14-dioxo-10-(2-aza-1-oxobuthyl)-2,3; 17,18-dinaphtho-cyclooctadecane (11)

This larite was prepared from the reaction of $\mathbf{3}$ and ethylisocyanate according to the general procedure afforded $\mathbf{1 1}$ in $91 \%$ yield and melting point of $184-185^{\circ} \mathrm{C}$. IR (KBr): 3391, 3294, 3055, 2925, 1678, 1542, 1503, 1432, 1265, 1236, 1089, 1076, $814 \mathrm{~cm}^{-1} ;{ }^{1} \mathrm{H}$ NMR (300 MHz, DMSO-d $\left.{ }_{6}\right) \delta: 3.37-3.39(\mathrm{~m}, 4 \mathrm{H}), 3.76(\mathrm{dd}, 4 \mathrm{H}$, $\mathrm{J}=3,6 \mathrm{~Hz}), 4.06(\mathrm{dd}, 2 \mathrm{H}, \mathrm{J}=3,6 \mathrm{~Hz}), 4.50(\mathrm{~s}, 4 \mathrm{H}), 4.94(\mathrm{dd}, 1 \mathrm{H}, \mathrm{J}=2,10 \mathrm{~Hz})$, $5.03(\mathrm{dd}, 1 \mathrm{H}, \mathrm{J}=3,16 \mathrm{~Hz}), 5.70-5.81(\mathrm{~m}, 1 \mathrm{H}), 7.32-7.42(\mathrm{~m}, 6 \mathrm{H}), 7.61(\mathrm{t}, 1 \mathrm{H}$, $\mathrm{J}=6 \mathrm{~Hz}), 7.86-7.93(\mathrm{~m}, 6 \mathrm{H}), 8.27(\mathrm{dd}, 2 \mathrm{H}, \mathrm{J}=3,7 \mathrm{~Hz}) \mathrm{ppm} ;{ }^{13} \mathrm{C} \mathrm{NMR}(75 \mathrm{MHz}$, DMSO- $\left.\mathrm{d}_{6}\right) \delta: 169.90,155.60,130.85,130.66,129.94,128.55,128.48,127.96$, $125.38,125.16,124.99,119.95,113.74,112.92,68.60,68.11,53.00,49.90,48.49$, $39.24,37.55 \mathrm{ppm}$; MS (EI) $\mathrm{m} / \mathrm{z}: 501$ [initial macrocycle (3)] ${ }^{+}(4 \%), 500(2 \%), 483$ (2\%), 300 (8\%), 218 (6\%), 187 (9\%), 144 (100\%), 115 (62\%), 56(5\%), 28 (16\%); Anal. Cald. for $\mathrm{C}_{31} \mathrm{H}_{32} \mathrm{~N}_{4} \mathrm{O}_{5} \mathrm{~S}: \mathrm{C}, 65.02 \%$; H, 5.63\%; N, 9.78\%; Found : C, 65.05\%; $\mathrm{H}, 5.57 \%$; N, 9.84\%. 


\section{Results and Discussion}

Dihydroxy naphthosulfide (1) was prepared based on the reported procedure. ${ }^{18}$ Corresponding diester (2) $)^{19,20}$ and aza crown (3) were synthesized, according to the Figure 1, based on the published procedure ${ }^{20}$. Lariats were prepared from the reaction of 3 and isocyanates (isothiocyanates) in $\mathrm{CH}_{2} \mathrm{Cl}_{2}$ in the presence of $\mathrm{Et}_{3} \mathrm{~N}$ in room temperature for 24 hours. All of the products recrystallized in chloroform and their IR, ${ }^{1} \mathrm{H}$ NMR, ${ }^{13} \mathrm{C}$ NMR and Mass spectra were obtained and showed correct structures. Their elemental analyses showed desired proportions of elements.

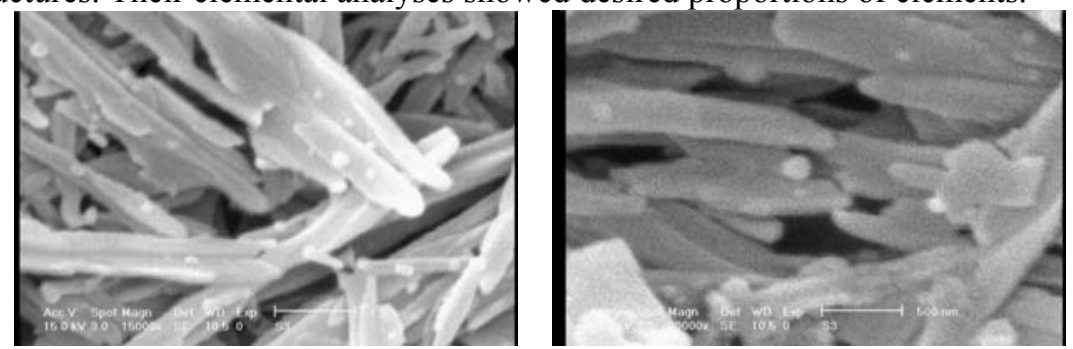

Figure 1. SEM images of 3

Scanning electron microscopy (SEM) images of macrocycles (3 and 8) were obtained and showed their structures in micro and nano scales as disc and fibrous structures (Schemes 1 and 2). Urea and thiourea functional groups in molecules spread the hydrogen bodings in the crystal structure and create flatten and rigid structures, these phenomena led to self-assemble and high organized structures ${ }^{21,22}$. Urea and thiourea groups interact with other molecules and in this conditions intramolecular hydrogen bonding is weaker than intermolecular hydrogen bondings ${ }^{21,22}$. According to the Schemes (1 and 2) phenyl as a side arm led to a flate and rigid structures and show the disc like structure of aggregated molecules. In all, the resonance between phenyl and thiourea led to a flatten structure in that region of molecule and in comparison between $\mathbf{3}$ and $\mathbf{8}$ because of the presence of this group, $\mathbf{8}$ is more flattened and rigid than $\mathbf{3}$ and intermolecular hydrogen bonding is stronger than $\mathbf{3}$. The more aggregated structures resulted from the stronger intermolecular hydrogen bonding and weaker intramolecular hydrogen bonding ${ }^{23}$. On the other hand, the phenyl group may lead to the $\pi-\pi$ interactions and more aggregations ${ }^{23}$.

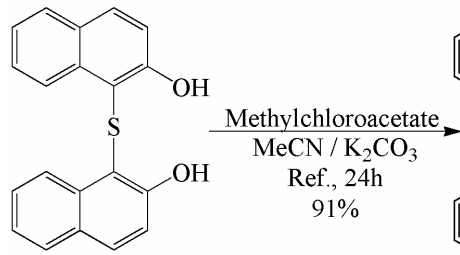

1<smiles>CC(=O)COc1ccc2ccccc2c1Sc1c(OCC(C)=O)ccc2ccccc12</smiles>

2

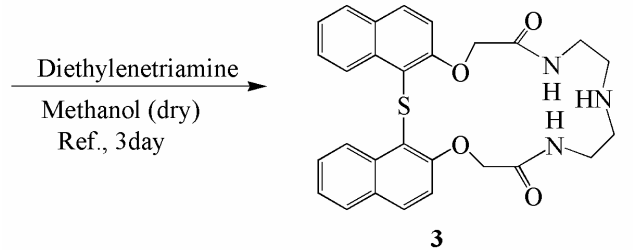

Scheme 1. Synthesis of macrocycle (3) 


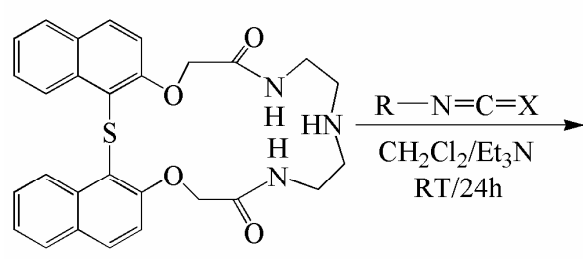

$$
\begin{array}{lll}
\text { Isothiocyanates: } & \mathrm{X}=\mathrm{S} & \mathrm{R}=\mathrm{Ph}(\mathbf{4}), \mathrm{Et}(\mathbf{5}) \\
\text { Isocyanates: } & \mathrm{X}=\mathrm{O} \quad \mathrm{R}=4-\mathrm{Cl}-\mathrm{Ph}(\mathbf{6}), \mathrm{Et}(\mathbf{7})
\end{array}
$$

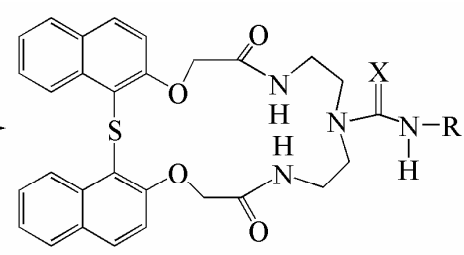

lariats: $\mathrm{X}=\mathrm{S} \quad \mathrm{R}=\mathrm{Ph}(\mathbf{8}), \mathrm{Et}$ (9) $\mathrm{X}=\mathrm{O} \quad \mathrm{R}=4-\mathrm{Cl}-\mathrm{Ph}(\mathbf{1 0}), \mathrm{Et}(\mathbf{1 1})$

Scheme 2. Synthesis of lariats (8-11)

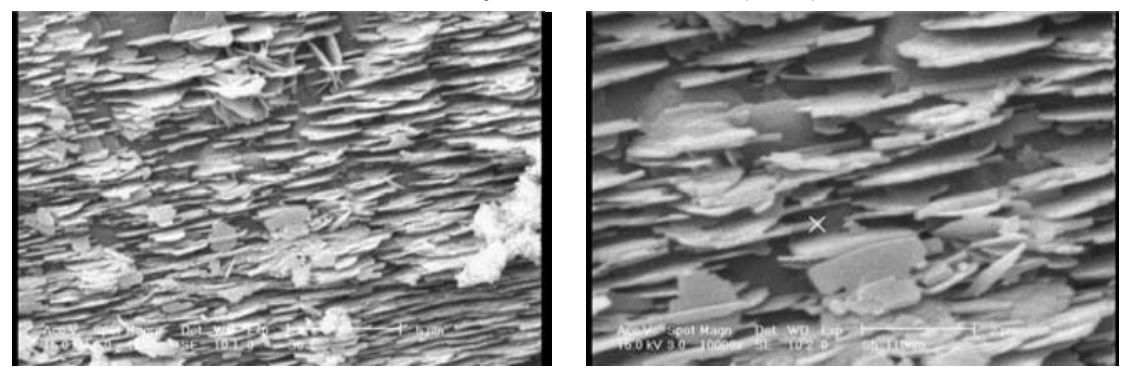

Figure 2. SEM images of 8

\section{References}

1. Pedersen C J, J Am Chem Soc., 1967, 89, 2495.

2. Pedersen C J, J Am Chem Soc., 1967, 89, 7017.

3. Christensen J J, Eatough D J, Izatt R M, Chem Rev., 1974, 74, 351-384.

4. a) Lauffer R B, Chem Rev., 1987, 87, 901-927; b) Späth A and König B, Molbank, 2010, M660.

5. Fabbrizzi L and Poggi A, Chem Soc Rev., 1995, 24, 197.

6. Even P and Boitrel B, Coord Chem Rev., 2006, 250, 519.

7. a) Lindoy L F, J Iran Chem Soc., 2004, 1, 1; b) Kim Y S, Wang F and Liu S, World J Hepatol, 2010, 2(1), 21-31.

8. Csokai V, Kadar M, Mai D L H, Varga O, Toth K, Kubinyi M, Grun A and Bitter I, Tetrahedron, 2008, 64(6), 1058.

9. a) Chartres J D, Lindoy L F and Meehan G V, Tetrahedron, 2006, 62, 4173;

b) Sharghi H, Khalifeh R and Salimi Beni A R, J Iran Chem Soc., 2010, 7, 275.

10. Satheeshkumar K S, Vasu G, Vishalakshi V, Moni M S and Jayakumar R, J Mol Recognit., 2004, 17(1), 67-75.

11. Fedorov Y V, Fedorova O A, Andryukhina E N, Shepel N E, Mashura M M, Gromov S P, Kuzmina L G, Churakov A V, Howard J A K, Marmois E, Oberle' J, Jonusauskas G and Alfimov M V, J Phys Org Chem., 2005, 18, 1032.

12. Tavano L, Muzzalupo R, Trombino S, Nicotera I, Rossi C O and La Mesa C, Colloids Surfaces B: Biointerfaces, 2008, 61(1), 30-38.

13. a) Liu H, Wang S, Luo Y, Tang W, Yu G, Li L, Chen C, Liu Y and Xi F, J Mater Chem., 2001, 11, 3063-3067; b) Piao M-H, Hwang J, Won M-S, Hyun M H and Shim Y-B, Electroanalysis, 2008, 20, 1293-1299; c) Jiang W and Schalley C A, Beilstein J Org Chem., 2010, 6(14). doi:10.3762/bjoc.6.14.

14. Aydogan A, Coady D J, Kim S K, Akar A, Bielawski C W, Marquez M and Sessler J L, Angew Chem., 2008, 120, 9794-9798. 
15. Ley C, Lacombat F, Plaza P, Martin M M, Leray I and Valeur B, Chem Phys Chem., 2009, 10, 276.

16. Koshkakaryan G, Parimal K, He J, Zhang X, Abliz Z, Flood A H and Liu Y, Chem Eur J., 2008, 14, 10211-10218.

17. Zhang J, Albelda M T, Liu Y and Canary J W, Chirality, 2005, 17(7), 404-420.

18. (a) Gump W S and Vitucci J C, J Am Chem Soc., 1945, 67, 238; (b) Gazdar M and Smiles S, J Chem Soc., 1910, 97, 2248.

19. Rostami E, Heidaryan D, Fattahi H, Shockravi A, Zarei M and Rakhshanderu F, Phosphorus, Sulfur Silicon, 2009, 184, 1924.

20. Shockravi A, Shamsipur M, Fattahi H, Taghdiri M, Heidaryan D, Alizadeh K, Rostami E, Abbaszadeh $\mathrm{M}$ and Yousefi A, J Inclusion Phenomena Macrocyclic Chem., 2008, 61(1-2), 153-160.

21. Ranganathan D, Lakshmi C and Karle I L, J Am Chem Soc., 1999, 121, 6103-6107.

22. Ranganathan D, Acc Chem Res., 2001, 34, 919.

23. a) Hartgerink J D, Zubarev E R, Stupp S I, Curr Opin Solid State Mater Sci., 2001, 5, 355-361; b) Zhang J, Albelda M T, LIU Y and Canary J W, Chirality, 2005, 17, 404-420; c) Joshi K B and Verma S, Tetrahedron, 2007, 63, 5602-5607. 


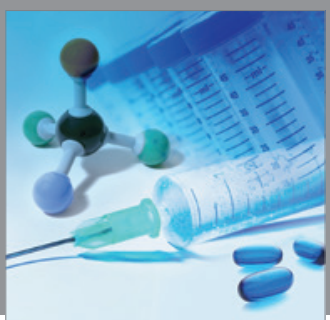

International Journal of

Medicinal Chemistry

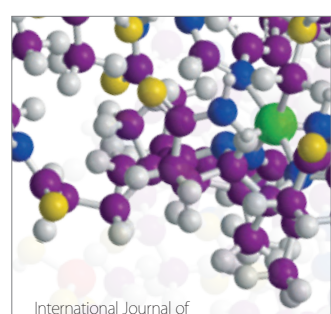

Carbohydrate Chemistry

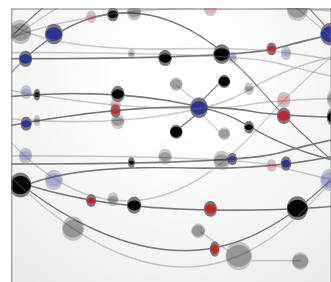

The Scientific World Journal
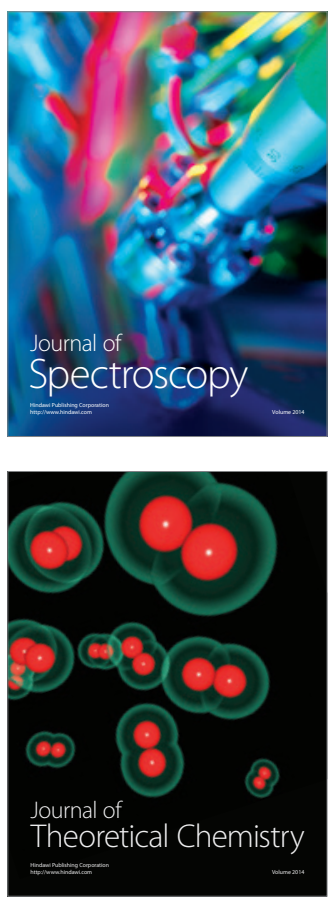
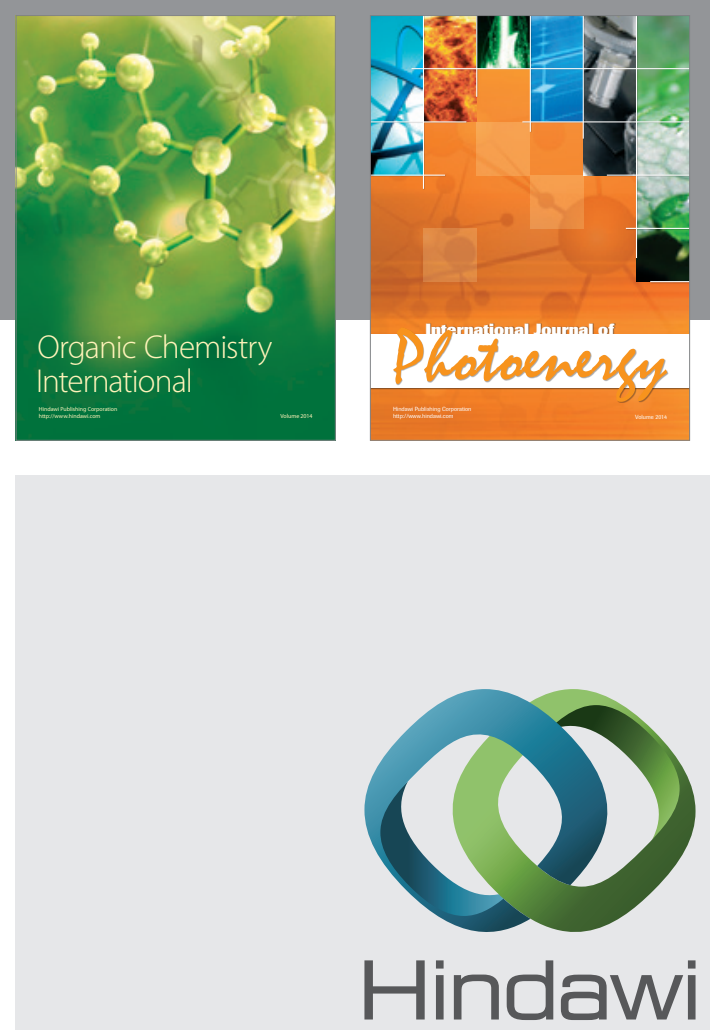

Submit your manuscripts at

http://www.hindawi.com
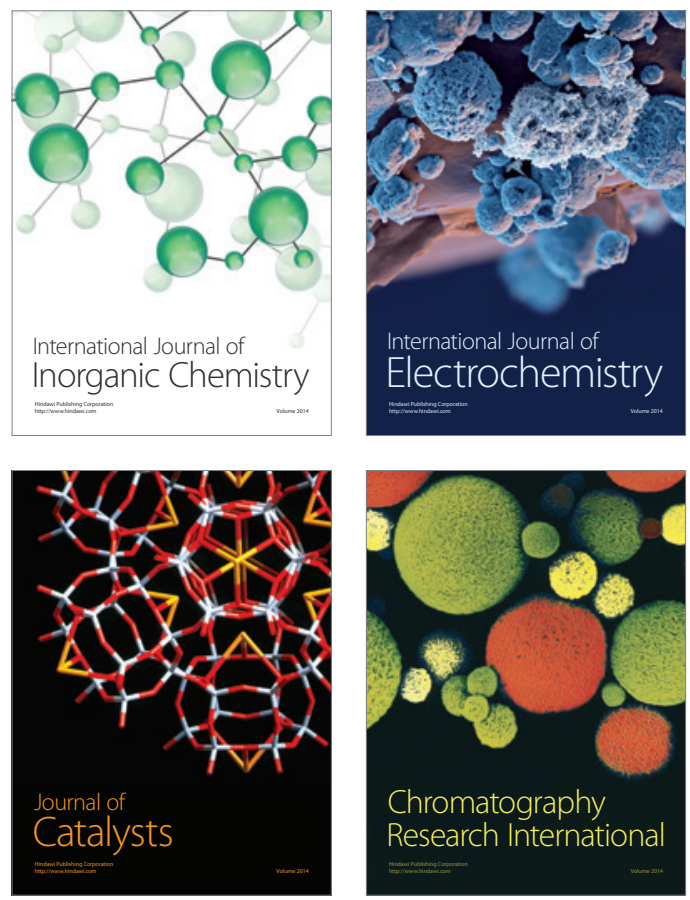
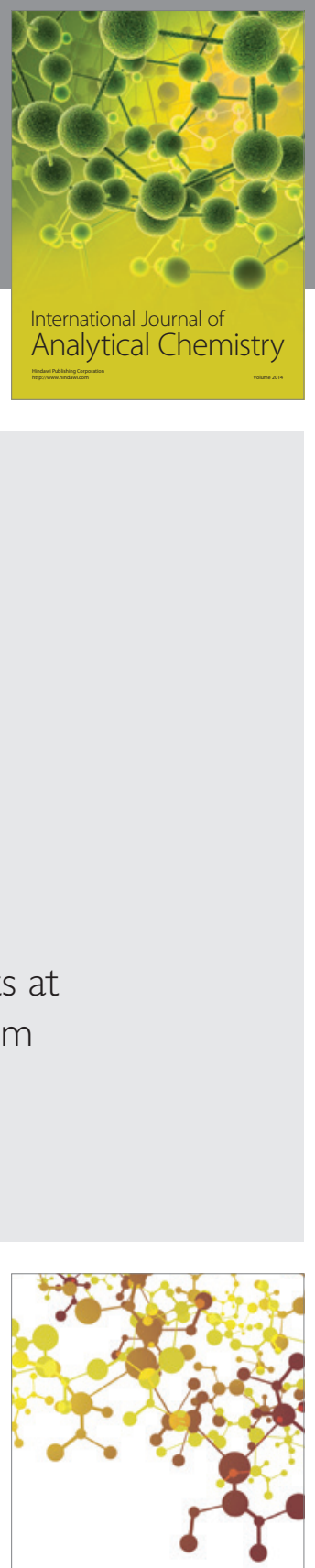

Journal of

Applied Chemistry
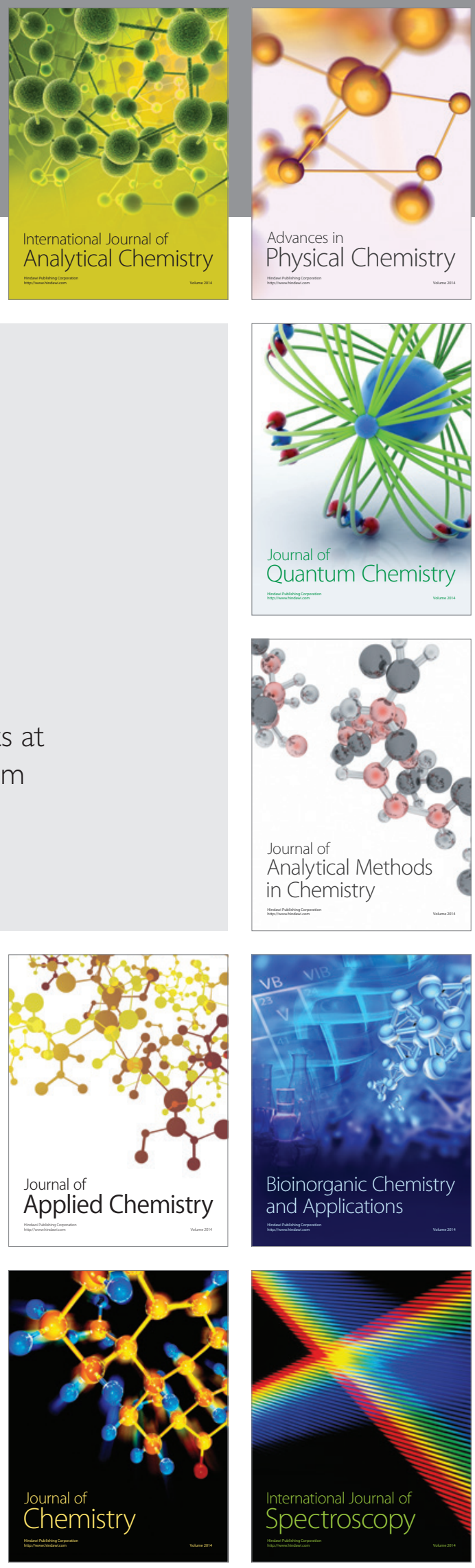